\title{
Multilevel analysis of factors that influence overweight in children: research in schools enrolled in northern Brazil School Health Program
}

Renata Andrade de Medeiros Moreira ${ }^{1 *}$ D, Tiago Ricardo Moreira², Glauce Dias da Costa ${ }^{3}$, Luiza Carla Vidigal Castro ${ }^{3}$ and Rosângela Minardi Mitre Cotta ${ }^{3}$

\begin{abstract}
Background: The study evaluates children in schools that participate in the School Health Program in the Northern region of Brazil with the objective of assessing whether their schools interfered in the development of overweight/ obesity and how individual and school environment variables behave according to contextual analysis.

Methods: The analyses were carried out with 1036 children from 25 municipal public schools in Northern Brazil that participated in the School Health Program. We evaluated both individual characteristics and scholar environment through univariate and multivariate logistic regressions to identify which of these factors were related to overweight/obesity as well as the effect of varying such associations.

Results: The considered individuals had an median age of 8 years, being $54.9 \%$ female and $27.8 \%$ presenting overweight/obesity. In multivariate logistic regression, the overweight/obesity variance in schools was 0.386 (individual variables) and 0.102 (individual and school variables), explaining $23.7 \%$ of the variation, reduction of ICC and MOR. The Akaike Information Criterion between the models was reduced and the likelihood ratio indicated better adequacy of the latter model. The investigated children had a greater chance of developing overweight/ obesity when they performed $2+$ sedentary activities/day, depending on school location as well as whether or not candies were sold in the school surroundings. On the other hand, a lower chance of developing overweight/ obesity was identified in children that ate $5+$ meals/day and practiced dance at school.

Conclusion: We observed that the variables inherent to both individuals and schools favored the development of overweight/obesity in children. It is relevant that scholar curriculums incorporate healthy eating interventions and encourage body practices associated with policies that restrain the sale of ultra-processed food in schools as well as the development of intersectoral actions between education and health to control childhood obesity.
\end{abstract}

Keywords: Obesity, Food consumption, Physical activity, School surroundings, Nutritional education, Public health

\footnotetext{
* Correspondence: renatamoreira@uft.edu.br

'Curso de Nutrição, Câmpus de Palmas, Universidade Federal do Tocantins,

Quadra 109 Norte, Avenida NS 15, ALCNO-14, Bloco de Apoio Logístico e

Administrativo 1 (BALA1) $2^{\circ}$ andar, sala 19, Curso de Nutrição. Bairro, Plano

Diretor Norte, Palmas, Tocantins 77001-090, Brazil

Full list of author information is available at the end of the article
}

(c) The Author(s). 2020 Open Access This article is licensed under a Creative Commons Attribution 4.0 International License, which permits use, sharing, adaptation, distribution and reproduction in any medium or format, as long as you give appropriate credit to the original author(s) and the source, provide a link to the Creative Commons licence, and indicate if changes were made. The images or other third party material in this article are included in the article's Creative Commons licence, unless indicated otherwise in a credit line to the material. If material is not included in the article's Creative Commons licence and your intended use is not permitted by statutory regulation or exceeds the permitted use, you will need to obtain permission directly from the copyright holder. To view a copy of this licence, visit http://creativecommons.org/licenses/by/4.0/. The Creative Commons Public Domain Dedication waiver (http://creativecommons.org/publicdomain/zero/1.0/) applies to the data made available in this article, unless otherwise stated in a credit line to the data. 


\section{Background}

In 2016, childhood overweight and obesity reached 340 million (18.4\%) [1] worldwide, thus constituting serious public health problems that are associated with increased risk of developing chronic non-communicable diseases (NCDs) in adulthood [2, 3], impairing the healthcare system [2] and denoting one of the biggest challenges due to their interaction with other social health factors and determinants, such as urbanization and agriculture [4].

Among the risk factors for obesity, individual $[1,3,5]$ - such as poor nutrition, physical inactivity (e.g., watching TV and playing electronic games on computers, videogames, and mobile phones), and genetic $[3,5]$ and psychological conditions $[2,5]$ - and contextual - such as social $[1,3]$ (e.g., interactions with family, friends, and the community in general) $[2,5]$ and physical (e.g., housing, workplaces, restaurants, supermarkets, and schools) $[1,5]$ environments - elements stand out.

Therefore, it is important to implement effective public policies that address socioeconomic and commercial factors, as well as programs that promote and provide healthcare services $[4,6]$, enabling regular access to healthy food and physical activity [6]. This requires intersectoral involvement, including joint efforts on communication, commerce, urbanism, agriculture, health, and education $[1,6]$.

Some environments are potentially important for the development of actions to control childhood obesity, among which schools stand out for their capacity to (i) integrate educational behavior-change procedures relying upon critical thinking, (ii) address multiple components intended to integrate nutrition and physical activity via diet and school curriculum, (iii) have suitable areas for recreation and practice of regular sports, (iv) foster parental and community participation, and (v) restrain the commercialization of ultra-processed food within school surroundings [5, 7-10]. Indeed, schools are also where children spend most of their daytime, consume a significant part of their daily calories [2], and exercise the most $[5,9]$.

International documents $[9,11]$ reinforce the inclusion of interventions to encourage healthy eating and increased physical activity in schools. In this respect, Brazilian Ministries of Health and Education, through intersectoral policies, established the School Health Program (SHP), aiming to contribute to the education of public primary school network students through healthcare prevention, promotion, and attention actions, which include those that promote healthy eating and body practices, physical activity, and leisure in schools $[12,13]$ as well as that prevent childhood obesity [13] in public educational institutions to be carried out by schools' and Primary Health Care (PHC) professionals [12, 13].
The study evaluates children enrolled in schools that participate in the School Health Program in the Northern region of Brazil, to verify whether type of school interferes with the development of overweight /obesity as well as how individual and school environment variables behave according to contextual analysis.

\section{Methods}

\section{Participants}

This study was part of a project entitled "Effectiveness of actions to control childhood obesity by the SHP in Palmas, Tocantins". Palmas, Tocantins State capital, is located in Northern Brazil and is administratively divided into 3 regions. From its 44 municipal public schools, 39 include primary school from 1 st to 5 th grade $[14,15]$ with 22,333 students [16]. Out of these schools, 16 were full-time $(7 \mathrm{~h} /$ day) while 23 were part-time $(4 \mathrm{~h} /$ day) [14, 15], all agreeing with the SHP [13]. The inclusion criteria were (ii) being a second- or fourth-grade student at one of the Palmas municipal public schools in 2018 and (ii) being literate. The exclusion criteria were (i) not presenting regular school attendance, (ii) being on sick leave, (iii) to have been transferred from the institution during collection, or (iv) to have had a disease that prevented participation.

For sample calculation we used 38\% prevalence of overweight and obesity in children aged 5 to 10 years old in Northern Brazil, 95\% significance level, 5\% error, 50\% design effect for cluster sample, and the amount of students enrolled in the second or fourth grades according to the 2017 School Census. First, we randomly selected 25 schools, being representative for the municipality (64.1\%). Afterwards, we randomly selected children respecting the proportionality for each school year, gender, and municipal administrative region, in accordance with schools' records, totaling 1036 children. In average, 41.44 children (minimum: 9; maximum: 115) per school were evaluated.

\section{Data collection and analysis}

We considered the anthropometric measurements of weight and height according with the recommendations of the Brazilian Food and Nutrition Surveillance System $[17,18]$ and evaluated the World Health Organization (WHO) Body Mass Index for Age (BMI/A) curve with the help of WHO AnthroPlus [19] by z-score, and classified the nutritional state $[17,18]$. Waist circumference (WC) was measured according to Frisancho [20], and Waist height to ratio (WHtR) was calculated and considered as a cutoff point $\leq 0.5$ absence of cardiovascular risk (ACVR) and $>0.5$ presence of cardiovascular risk (PCVR) [21].

We performed the 6-min walking cardiorespiratory fitness test on a 30-m course to determine students' 
aerobic capacity proposed by the American Thoracic Society [22], and calculated the index walked distance/ height, both in meters $(\mathrm{T} 6 \mathrm{M} / \mathrm{t})$ according to Kain et al. [23]. We emphasize that the 6-min walk test (T6M) is a standard criterion regularly utilized in children, and presents validity [24-26] and reproducibility [24-27].

To evaluate food consumption and physical activity during the day before, we used the School Monitoring System of Food Consumption and Physical Activity [28] validated in Brazil for food consumption [29-31] and the evaluation of physical activity [32]. The reproducibility [33] of the system and its use as a Web-Based Questionnaire [34] were evaluated. We first advised on completing the questionnaire and supervised the process. We evaluated food intake considering the numbers of daily meals $[34,35]$ and food portions from each food group according with the Dietary Guidelines for the Brazilian Population [35]. The children had 32 food options available for each meal [21], considering 1 portion every time the food was reported [34]. The cut-off point of the categorical variables in relation to number of meals (5 portions/day) and portions of food groups were defined according to the guide [35]: (cereals: 6 portions/day, vegetables: minimum 3 portions/day, fruits: minimum 3 portions/day, legumes: 1 to 2 portions/day, milk and dairy products: minimum 3 portions/day, meat and egg: 2 portions/day, fats: maximum 1 portion/day and sugar: maximum 1 portion/day).

We analyzed the physical activities performed on the day before, with the possibility of choosing 32 activities, and the child's assimilation with the intensity to perform them, evaluating the percentage of active and non-active activities and the intensity perception score [34]. Given the lack of reference for adequacy cut-off point, the median value was utilized as categorical variables.

We applied a questionnaire with school heads about data pertinent to the type of school shift, number of enrolled students, schooling years offered; physical activities that were offered in addition to physical education class; school feeding (number of meals and cafeterias' condition); food sale around the institution; school garden (existence and types of grown food); and food and nutrition education actions and body practices outlined in the SHP [13].

\section{Statistical analysis}

We defined as dichotomous dependent variable the nutritional status classification according to BMI/A, with the categories thinness/eutrophy (0) and overweight/ obesity [1]. As explanatory variables we included in level 1 those relating to individual children's data and in level 2 contextual characteristics related to schools. Numerical variables that deviate from normality were transformed into categorical variables based on cutoff points in the literature, in the absence of cutoff points, median values were utilized. This definition was adopted due to the absence of a normal distribution after the logarithmization of the variables.

In the initial analysis we described categorical variables using absolute numbers and percentages, while continuous variables were described by median and $95 \%$ confidence interval $(95 \% \mathrm{CI})$. We performed Pearson's chisquared test and Student's t-test to estimate the association between nutritional status and individual and school characteristics. The strength of the association between nutritional status and explanatory variables was assessed using the odds ratio (OR) and their respective $95 \% \mathrm{CI}$ using bivariate and multivariate multilevel logistic regressions.

To identify the mean association between individual and contextual (school environment) health variables for neighborhood clusters (schools), multilevel logistic regression was utilized and the results were expressed in OR and their respective 95\%CI. The individual and contextual variables were entered using a forward stepwise method assessed with the Wald test. For the multilevel analysis of individual heterogeneity, we adopted a combination of specific contextual effect (SCE), evaluated by OR and 95\%CI and general contextual effect (GCE) evaluated by Intra-Class Correlation Coefficient (ICC), mean odds ratio (MOR) and area under the receiver operating characteristic curve (AUC) [36, 37].

The SCE presented as OR estimates the degree of association between the specific characteristics of the neighborhood (school environment) and the individual results under investigation (classification of nutritional status). It demonstrates the mechanisms mediating GCE, possibly drawing a contradictory conclusion that the general context is relevant when it is not. Therefore, SCE analysis was performed in conjunction with GCE [37], which evaluates the effect of the cluster on individual results [38].

GCE estimates the effects of neighborhood contexts on individual results without referring to specific characteristics of the neighborhood [37] through measures of variation components (ICC and AUC), and of heterogeneity (MOR) [38]. General contextual effects were estimated by ICC as it is a measure of discriminatory precision which depends on the variation of cluster-specific random effect distribution [36, 38], thus required for hierarchical structures [38]. The ICC quantifies the size of the GCE, considering the context as the most relevant for clarifying the differences in individual results, especially because schools are defined by geographical delimitations that do not capture the relevant physical or sociological contexts that influence an individual's health [37].

Because the ICC for binary responses is based on the latent response of the model and the variance of the 
regression is defined by the log-odds scale, we adopted MOR heterogeneity analysis to estimate GCE [36] in terms of level of variation or heterogeneity between clusters [38]. In other words, MOR allows one to quantify the contextual effect on the same scale applied for the measures of association as well quantify whether the effect at the individual level would covert the outcome's probabilities [38]. We calculated MOR to estimate the contextual effect, i.e., to quantify the variation between schools comparing two children with the same covariates from two different, randomly chosen schools [39]. Therefore, MOR takes into account higher and lower overweight-prone children, quantifying the variance of the scholar environment level in terms of OR, being comparable to the fixed effects OR and providing a heterogeneity measurement scale [39-41].

We also evaluated the AUC since it is a measure of the model's discriminatory precision to compare individuals correctly based on predicted individual probabilities [36]. In other words, the AUC compares all possible pairs of individuals who have suffered excess weight/ obesity and a subject without no prior history, being the statistics showing the proportion of individuals who experienced overweight/obesity were more likely to experience the same event than an individual with no prior history [38].

The AUC is a graphical representation of the rate of true positives (FPV) or sensitivity, in relation to the rate of false positives (FPF), specificity, for different thresholds of binary classifications of the predicted probabilities. It has values between 1 and 0.5 , where 1 represents perfect discrimination and 0.5 represents a covariate with no predictive value [36, 38].

For the univariate logistic regression, we analyzed the OR and $95 \% \mathrm{CI}$ of nutritional status with the individual variables and the school environment adjusted for child's school. For multilevel logistic regression we first adjusted a null model without explanatory variables to verify the significance of the nutritional status variance among schools (model I). Then we performed to test, by bivariate analysis, the individual variables of the child (level 1) with nutritional status. Subsequently, we performed model II, adjusting the multivariate model for the individual-level explanatory variables that presented $p<0.20$ in the bivariate analysis and maintained those with $p<0.05$ [38]. We inserted 7 individual variables: $\mathrm{T} 6 \mathrm{M}$, adequacy of number of daily meals, classification of daily consumption of meat, fat and sugar, classification of number of daily sedentary and non-sedentary activities.

In Model III, we included 14 variables relevant to the school environment (level 2: school administrative region, school shift, taking dance classes and body practice at school, number of physical activity classes offered at school, number of meals offered by school, sale of food in the school environment, sale of fried savory snacks, sweets, sugary drinks, existence of school garden, nutritional assessment carried out by school and PHC, actions taken by school to prevent childhood obesity) coupled with the 3 variables that remained on Model II [38], keeping the same statistical criteria. To verify the model settings, we used the Akaike Information Criterion (AIC) and likelihood ratio test. Statistical analyses were carried out on STATA software, version 13.0.

\section{Results}

The median age of the children was 8.0 years, being $54.9 \%$ female and $51.4 \%$ second-grade students. We identified that the BMI/A was 0.40 ; $95 \% \mathrm{CI}: 0.13$ to 0.30 , considering that $72.2 \%$ presented thinness/eutrophy (0.37; $95 \%$ CI: -0.50 to 0.37 ) and $27.8 \%$ were overweight/ obesity $(1.74 ; 95 \% \mathrm{CI}: 1.80$ to 2.00$)$. We observed that WC (thinness /eutrophy: 55.0; 95\%CI: 55.0 to 55.6 vs. overweight/obesity: 66.0 ; $95 \% \mathrm{CI}$ : 66.7 to $68.7 ; p \leq 0.001$ ), WHtR (thinness/eutrophy: 0.42; 95\%CI: 0.41 to 0.42 vs. overweight /obesity: 0.48 ; $95 \% \mathrm{CI}$ : 0.49 to $0.50 ; \mathrm{p} \leq 0.001$ ) and cardiovascular risk (ACVR: thinness/eutrophy: 99.9\% vs. overweight/obesity: 61.5\%; PCVR: thinness/eutrophy: $0.1 \%$ vs. overweight / obesity: 38.5$)$ were higher in overweight children (Table 1).

The characteristics of the studied children and schools are described in Table 1. Comparisons between these characteristics and the nutritional status classification are shown in Table 2. The median $\mathrm{T} 6 \mathrm{M} / \mathrm{t}$ was lower in overweight/obesity children, as $65.3 \%$ of these performed $2+$ sedentary activities on the previous day. As for food consumption, $38.2 \%$ of the children consumed more sugar that the recommended level, consumption which had a prevalence of $43.4 \%$ in those who were overweight/obesity.

Regarding the school characteristics, we observed that $44.3 \%$ of the children with thinness/eutrophy were from Southern Palmas and $35.1 \%$ of those who were overweight/obesity were from the Center-South. From the overweight/obesity children, we highlight that $49.3 \%$ studied part-time, $52.8 \%$ performed less than three physical activities/week at school, and 49.7 and $48.6 \%$ did not participate in dance or body practice classes, respectively.

Also regarding overweight/obesity children, we found that $61.1 \%$ had only one meal at their schools, $48.3 \%$ had either no cafeteria or inadequate cafeteria at their schools, and $91.0 \%$ attended schools that did not sell natural, fresh juice in their surroundings, $94.0 \%$ of which sold candies. The absence of school gardens (49.7\%), as well as the non-cultivation of green leafy vegetables $(49.7 \%)$, legumes $(69.1 \%)$, and tubers $(88.2 \%)$, was more present in institutions with higher prevalence of overweight/obesity in children. We also found that $96.7 \%$ of 
Table 1 Characteristics of children and municipal public schools in Northern Brazil, according to the BMI classification by age, 2018. $N=1036$

\begin{tabular}{|c|c|c|c|c|}
\hline \multirow[t]{2}{*}{ Variables $^{\mathbf{a}}$} & \multicolumn{2}{|l|}{ BMI Classification by Age } & \multirow[t]{2}{*}{ Total } & \multirow[t]{2}{*}{$\boldsymbol{p}$ Value } \\
\hline & Thinness and Eutrophy & Overweight and Obesity & & \\
\hline \multicolumn{5}{|l|}{ Gender } \\
\hline Female & $405(54.1 \%)$ & $164(56.9 \%)$ & $569(54.9 \%)$ & \multirow[t]{2}{*}{$0.417^{b}$} \\
\hline Male & $343(45.9 \%)$ & $124(43.1 \%)$ & $467(45.1)$ & \\
\hline Age & $8.0(8.5-8.7)$ & $8.6(8.5-8.7)$ & $8.0(8.5-8.7)$ & $0.943^{c}$ \\
\hline \multicolumn{5}{|l|}{ Grade } \\
\hline Second & 389 (52\%) & $144(50 \%)$ & $533(51.4 \%)$ & \multirow[t]{2}{*}{$0.563^{b}$} \\
\hline Fourth & $359(48 \%)$ & $144(50 \%)$ & $503(48.6 \%)$ & \\
\hline \multicolumn{5}{|l|}{ Anthropometric Variables } \\
\hline Waist Circumference & $55.0(55.0-55.6)$ & $66.0(66.7-68.7)$ & $57.0(58.3-59.3)$ & $\leq 0,001^{d}$ \\
\hline Waist-to-height ratio & $0.42(0.41-0.42)$ & $0.48(0.49-0.50)$ & $0.43(0.43-0.44)$ & $\leq 0,001^{d}$ \\
\hline \multicolumn{5}{|l|}{ Classification of Waist-to-heigh ratio } \\
\hline Absence of cardiovascular risk & $732(99,9 \%)$ & $174(61,5 \%)$ & $906(89,2 \%)$ & \multirow[t]{2}{*}{$\leq 0,001^{b}$} \\
\hline Presence of cardiovascular risk & $1(0,1 \%)$ & $109(38,5 \%)$ & $110(10,8 \%)$ & \\
\hline \multicolumn{5}{|l|}{ Physical Activity } \\
\hline Covered distance divided by height & $343.8(342.6-350.1)$ & $332.5(329.1-341.4)$ & $340.8(340.1-346.5)$ & $0.003^{c}$ \\
\hline Number of non-sedentary activities & $2.0(2.2-2.5)$ & $2.0(2.1-2.6)$ & $2.0(2.2-2.5)$ & $0.951^{c}$ \\
\hline \multicolumn{5}{|l|}{ Non-sedentary activities classification } \\
\hline$<$ Median (<2 activities) & $264(35.3 \%)$ & $114(39.6 \%)$ & $378(36.5 \%)$ & \multirow[t]{2}{*}{$0.199^{b}$} \\
\hline$\geq$ Median ( $\geq 2$ activities) & $484(64.7 \%)$ & $174(60.4 \%)$ & $658(63.5 \%)$ & \\
\hline Non-sedentary activities intensity & $6.0(6.2-7.0)$ & $5.0(5.7-7.0)$ & $5.0(6.2-6.9)$ & $0.447^{c}$ \\
\hline \multicolumn{5}{|c|}{ Non-sedentary activities intensity classification } \\
\hline$<$ Median (<5 activities) & $322(43 \%)$ & $137(47.6 \%)$ & $459(44.3 \%)$ & \multirow[t]{2}{*}{$0.189^{b}$} \\
\hline$\geq$ Median ( $\geq 5$ activities) & $426(57 \%)$ & $151(52.4 \%)$ & $577(55.7 \%)$ & \\
\hline Number of sedentary activities/day & $2.0(2.1-2.4)$ & $2.0(2.5-3.0)$ & $2.0(2.3-2.6)$ & $0.005^{c}$ \\
\hline \multicolumn{5}{|l|}{ Sedentary activities classification } \\
\hline$<$ Median ( $<2$ activities) & $331(44.3 \%)$ & $100(34.7 \%)$ & $431(41.6 \%)$ & \multirow[t]{2}{*}{$0.005^{\mathrm{b}}$} \\
\hline$\geq$ Median ( $\geq 2$ activities) & $417(55.7 \%)$ & $188(65.3 \%)$ & $605(58.4 \%)$ & \\
\hline \multicolumn{5}{|l|}{ Food Consumption } \\
\hline Number of meals & $5.0(4.7-4.8)$ & $5.0(4.6-4.9)$ & $5.0(4.7-4.8)$ & \multirow[t]{4}{*}{$0.909^{c}$} \\
\hline \multicolumn{4}{|l|}{ Meals adequacy } & \\
\hline Insufficient (<5 meals) & $276(36.9 \%)$ & $115(39.9 \%)$ & $391(37.7 \%)$ & \\
\hline Adequate ( $\geq 5$ meals) & $472(63.1 \%)$ & $173(60.1 \%)$ & $645(62.3 \%)$ & \\
\hline Cereals group portions & $3.0(2.8-3.1)$ & $3.0(2.7-3.1)$ & $3.0(2.8-3.0)$ & $0.605^{c}$ \\
\hline \multicolumn{5}{|l|}{ Cereals group adequacy } \\
\hline Insufficient (< 6 portions) & $706(94.4 \%)$ & $276(95.8 \%)$ & $982(94.8 \%)$ & \multirow[t]{2}{*}{$0.347^{\mathrm{b}}$} \\
\hline Adequate ( $\geq 6$ portions) & $42(5.6 \%)$ & $12(4.2 \%)$ & $54(5.2 \%)$ & \\
\hline Vegetables group portions & $0.0(0.6-0.8)$ & $0.0(0.6-0.8)$ & $0.0(0.6-0.8)$ & $0.923^{c}$ \\
\hline \multicolumn{5}{|l|}{ Vegetables group adequacy } \\
\hline Insufficient (<3 portions) & $713(95.3 \%)$ & $274(95.1 \%)$ & $987(95.3 \%)$ & \multirow[t]{2}{*}{$0.902^{b}$} \\
\hline Adequate ( $\geq 3$ portions) & $35(4.7 \%)$ & $14(4.9 \%)$ & $49(4.7 \%)$ & \\
\hline Fruits group portions & $1.0(0.9-1.1)$ & $1.0(1.0-1.3)$ & $1.0(1.0-1.1)$ & $0.087^{c}$ \\
\hline
\end{tabular}


Table 1 Characteristics of children and municipal public schools in Northern Brazil, according to the BMl classification by age, 2018. $N=1036$ (Continued)

\begin{tabular}{|c|c|c|c|c|}
\hline \multirow[t]{2}{*}{ Variables $^{\mathbf{a}}$} & \multicolumn{2}{|l|}{ BMI Classification by Age } & \multirow[t]{2}{*}{ Total } & \multirow[t]{2}{*}{$\boldsymbol{p}$ Value } \\
\hline & Thinness and Eutrophy & Overweight and Obesity & & \\
\hline \multicolumn{5}{|l|}{ Fruits group adequacy } \\
\hline Insufficient (<3 portions) & $662(88.5 \%)$ & $249(86.5 \%)$ & $911(87.9 \%)$ & \multirow[t]{2}{*}{$0.365^{b}$} \\
\hline Adequate ( $\geq 3$ portions) & $86(11.5 \%)$ & 39 (13.5\%) & $125(12.1 \%)$ & \\
\hline Dairy group portions & $0.0(0.7-0.8)$ & $0.0(0.6-0.8)$ & $0.0(0.7-0.8)$ & $0.866^{c}$ \\
\hline \multicolumn{5}{|l|}{ Dairy group adequacy } \\
\hline Insufficient (<3 portions) & 708 (94.7\%) & $270(93.8 \%)$ & 978 (94.4\%) & \multirow[t]{2}{*}{$0.571^{b}$} \\
\hline Adequate (3 portions) & $40(5.3 \%)$ & $18(6.3 \%)$ & $58(5.6 \%)$ & \\
\hline Meat and eggs group portions & $2.0(1.5-1.7)$ & $2.0(1.5-1.8)$ & $2.0(1.5-1.7)$ & $0.666^{c}$ \\
\hline \multicolumn{5}{|l|}{ Meat and eggs group adequacy } \\
\hline Insufficient (< 1 portions) & $118(15.8 \%)$ & $34(11.8 \%)$ & $152(14.7 \%)$ & \multirow[t]{3}{*}{$0.233^{b}$} \\
\hline Adequate (1-2 portions) & $498(66.7 \%)$ & $205(71.2 \%)$ & $703(67.9 \%)$ & \\
\hline Excessive (> 2 portions) & $131(17.5 \%)$ & $49(17 \%)$ & $180(17.4 \%)$ & \\
\hline Legume group portions & $1.0(1.2-1.3)$ & $1.0(1.0-1.2)$ & $1.0(1.2-1.3)$ & $0.065^{c}$ \\
\hline \multicolumn{5}{|l|}{ Legume group adequacy } \\
\hline Insufficient (<1 portions) & $179(23.9 \%)$ & $76(26.4 \%)$ & $255(24.6 \%)$ & \multirow[t]{3}{*}{$0.469^{b}$} \\
\hline Adequate (1-2 portions) & $526(70.3 \%)$ & $200(69.4 \%)$ & $726(70.1 \%)$ & \\
\hline Excessive (> 2 portions) & $43(5.7 \%)$ & $12(4.2 \%)$ & $55(5.3 \%)$ & \\
\hline Fat group portions & $1.0(1.5-1.7)$ & $1.0(1.5-1.8)$ & $1.0(1.5-1.7)$ & $0.538^{c}$ \\
\hline \multicolumn{5}{|l|}{ Fat group adequacy } \\
\hline Adequate ( $\leq 0$ portions) & $216(28.9 \%)$ & $71(24.7 \%)$ & $287(27.7 \%)$ & \multirow[t]{2}{*}{$0.173^{b}$} \\
\hline Excessive ( $\geq 1$ portions) & $532(71.1 \%)$ & $217(75.3 \%)$ & 749 (72.3\%) & \\
\hline Sugar group portions & $1.0(1.1-1.3)$ & $(1.0-1.3-1.7)$ & $1.0(1.2-1.4)$ & $0.019^{c}$ \\
\hline \multicolumn{5}{|l|}{ Sugar group adequacy } \\
\hline Adequate ( $\leq 0$ portions) & $477(63.8 \%)$ & $163(56.6 \%)$ & $640(61.8 \%)$ & \multirow[t]{2}{*}{$0.033^{\mathrm{b}}$} \\
\hline Excessive ( $\geq 1$ portions) & $271(36.2 \%)$ & $125(43.4 \%)$ & $396(38.2 \%)$ & \\
\hline
\end{tabular}

Note: BMI Body Mass Index; ${ }^{a}$ Numbers with percentages or medians with $95 \%$ confidence interval; ${ }^{b}$ Chi-squared test; ${ }^{\mathrm{c}}$ Student's t-test; ${ }^{\mathrm{d}} \mathrm{Mann}$-Whitney

overweight/obesity children attended schools that did not use nutritional assessment to plan actions for foodrelated/nutritional education, $42.7 \%$ attended schools that did not hold the Food Week, and only 36.8\% attended schools in which the nutritional status assessment was performed by reference PHC professionals.

Table 3 shows the outcome of the multilevel logistic regression analysis. In the bivariate analysis, we found that the chance of being overweight/obesity was lower in children who (i) studied in Southern Palmas, were enrolled in full-time schools, had early and late primary education, and had pre-school and early and late primary educations; (i)) had higher T6M/t index, had dance and body practice classes, performed $3+$ physical activity classes/week; (iii) consumed three meals during the school period and had a school garden and access to nutritional assessment by the school. However, performing $2+$ sedentary activities/day, consuming $1+$ portion of sugar/day, and studying in a school that sold fried snacks and candies in its surroundings and at which the qualified PHC performed a nutritional status assessment increased the chances of a child presenting overweight/ obesity.

In model I, we verified the nutritional status variance between schools $\left(\sigma^{2}: 0.411 ; 95 \% \mathrm{CI}: 0.221-0.674\right)$ with MOR of 1.48; in other words, differences between schools can increase by $48 \%$ the individual chances of being overweight/obesity, and ICC of $4.88 \%$, which meant that $4.88 \%$ of the total variation in overweight/obesity among the children is due to individual variables. In model II, we identified that the individual variables that remained independently associated with the increased chance of being overweight/obesity were performing $2+$ sedentary activities/day and consuming 1+ portion of the sugar group/ day, while consuming $5+$ meals/day was associated with a lower chance of being overweight/obesity.

In model III, by inserting the contextual level variables, we observed that the chances of developing overweight/ 
Table 2 Characteristics of municipal public schools in Northern Brazil, according to the BMI/A of the evaluated children, 2018. $\mathrm{N}=$ 1036

\begin{tabular}{|c|c|c|c|c|}
\hline Variables & Thinness and Eutrophy & Overweight and Obesity & Total & $\mathrm{p}$ Value \\
\hline \multicolumn{5}{|l|}{ General Characteristics } \\
\hline \multicolumn{5}{|l|}{ Administrative region } \\
\hline North & $172(23.0 \%)$ & $98(34.0 \%)$ & $270(26.1 \%)$ & \multirow[t]{3}{*}{$\leq 0.001$} \\
\hline Center-South & $245(32.8 \%)$ & $101(35.1 \%)$ & $346(33.4 \%)$ & \\
\hline South & $331(44.3 \%)$ & 89 (30.9\%) & 420 (40.5\%) & \\
\hline \multicolumn{5}{|l|}{ School shift } \\
\hline Part-time & $287(38.4 \%)$ & $142(49.3 \%)$ & $429(41.4 \%)$ & \multirow[t]{2}{*}{$0.001^{b}$} \\
\hline Full-time & $461(61.6 \%)$ & $146(40.7 \%)$ & 607 (58.6\%) & \\
\hline Number of enrolled students & $728.0(738.5-778.2)$ & $711.4(679.8-741.6)$ & $697.0(728.3-761.8)$ & $0.011^{c}$ \\
\hline
\end{tabular}

Physical activities offered

Dance

No $287(38.4 \%) \quad 143(49.7 \%)$

$145(50.3 \%)$

$430(41.5 \%)$

$0.001^{b}$

Yes

$461(61.6 \%)$

$606(58.5 \%)$

Body Practices

No

294 (39.3\%)

140 (48.6\%)

$434(41.9 \%)$

$0.007^{\mathrm{b}}$

Yes

$454(60.7 \%)$

$148(51.4 \%)$

$602(58.1 \%)$

Weekly physical activity

$\begin{array}{ll}<3 \text { classes } & 312(41.7 \%) \\ \geq 3 \text { classes } & 436(58.3 \%)\end{array}$

$152(52.8 \%)$

$464(44.8 \%)$

$0.001^{b}$

School feeding

Number of meals

$1 \mathrm{meal} /$ day

368 (49.2\%)

$176(61.1 \%)$

544 (52.5\%)

$0.001^{\mathrm{b}}$

3 meals/day

$380(50.8 \%)$

$112(38.9 \%)$

$492(47.5 \%)$

Cafeteria at school

Absent or inadequate

297 (39.7\%)

139 (48.3\%)

$436(42.1 \%)$

$0.012^{b}$

Adequate

$451(60.3 \%)$

$149(51.7 \%)$

600 (57.9\%)

\section{Food sale in school surroundings}

Food outlets

No $125(16.7 \%)$

$35(12.2 \%)$

$160(15.4 \%)$

$0.069^{b}$

Yes

$253(87.8 \%)$

$876(84.6 \%)$

Juices and soft drinks sale

No

$645(86.2 \%)$

$262(91.0 \%)$

$907(87.5 \%)$

$0.038^{b}$

Yes

$103(13.8 \%)$

$26(9.0 \%)$

$129(12.5 \%)$

Sweetened beverages sale

No

Yes

$350(46.8 \%)$

$398(53.2 \%)$

Fried snacks sale

No

Yes

Processed snacks sale

No

Yes

Candy sale
$338(45.2 \%)$

410 (54.8\%)

382 (51.1\%)

366 (48.9\%)
146 (50.7\%)

142 (49.3\%)

153 (53.1\%)

135 (46.9\%)

138 (47.9\%)

$150(52.1 \%)$
496 (47.9\%)

$0.260^{b}$

540 (52.1\%)

491 (47.4\%)

$0.022^{b}$

545 (52.6\%)

520 (50.2\%)

$0.363^{b}$

516 (49.8\%) 
Table 2 Characteristics of municipal public schools in Northern Brazil, according to the BMI/A of the evaluated children, 2018. $\mathrm{N}=$ 1036 (Continued)

\begin{tabular}{cllll}
\hline Variables & Thinness and Eutrophy & Overweight and Obesity & Total & p Value \\
\hline No & $173(23.1 \%)$ & $46(16.0 \%)$ & $219(21.1 \%)$ & $0.011^{b}$ \\
Yes & $575(76.9 \%)$ & $242(84.0 \%)$ & $817(78.9 \%)$
\end{tabular}

\section{School garden}

Garden for school feeding

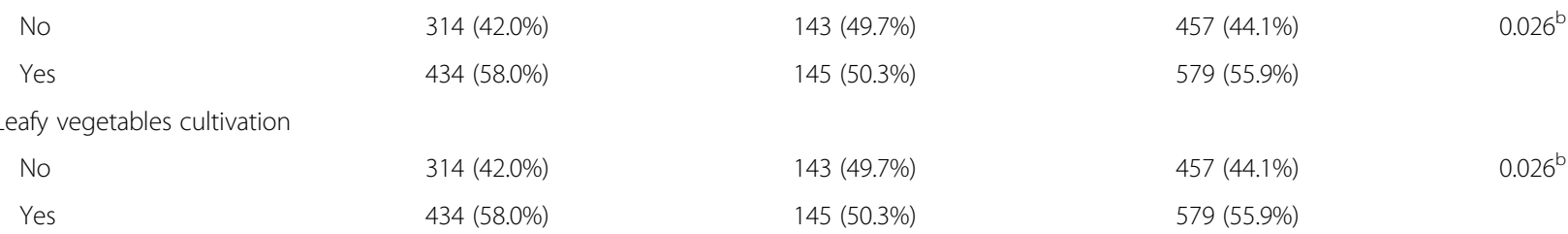

Legumes cultivation

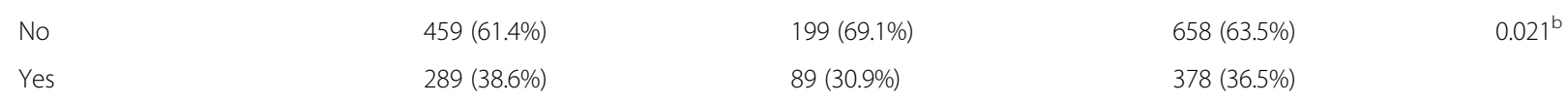

Tuber cultivation

No $\quad 615(82.2 \%)$

Yes

$133(17.8 \%)$

\section{SHP actions performed at school}

Nutritional state assessment

No $659(88.1 \%)$

Yes

$89(11.9 \%)$

Healthy eating promotion

No

$536(71.7 \%)$

$212(28.3 \%)$

$254(88.2 \%)$

$34(11.8 \%)$

$869(83.9 \%)$

$167(16.1 \%)$

Yes

Childhood obesity prevention

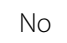

Yes

$718(96.0 \%)$

$30(4.0 \%)$

Health Week at school

$\begin{array}{ll}\text { No } & 388(51.9 \%) \\ \text { Yes } & 360(48.1 \%)\end{array}$

Science Fair

No $\quad 37(4.9 \%)$

Yes

$711(95.1 \%)$

Held Food Week
No
$379(50.7 \%)$
Yes

$369(49.3 \%)$
Health Care

\section{SHP actions performed in the Primary Health Care}

Nutritional state assessment

\begin{tabular}{|c|c|c|c|c|}
\hline No & $535(71.5 \%)$ & $182(63.2 \%)$ & 717 (69.2\%) & $0.009^{b}$ \\
\hline Yes & $213(28.5 \%)$ & $106(36.8 \%)$ & $319(30.8 \%)$ & \\
\hline \multicolumn{5}{|c|}{ lealthy eating promotion } \\
\hline No & $519(69.4 \%)$ & $211(73.3 \%)$ & $730(70.5 \%)$ & $0.220^{b}$ \\
\hline Yes & $229(30.6 \%)$ & $77(26.7 \%)$ & 306 (29.5\%) & \\
\hline
\end{tabular}

Note: ${ }^{\mathrm{a} N u m b e r s}$ with percentages or medians with $95 \%$ confidence interval; ${ }^{\mathrm{b}}$ Chi-squared test; ${ }^{\mathrm{C}}$ Student's t-test. BMI/A Body Mass Index for Age
898 (86.7\%)

$0.868^{b}$

$49(17.0 \%)$

$138(13.3 \%)$

206 (71.5\%)

742 (71.6\%)

$0.967^{b}$

$82(28.5 \%)$

$294(28.4 \%)$

$283(98.3 \%)$

1001 (96.6\%)

$0.069^{b}$

$5(1.7 \%)$

$136(47.2 \%)$

$524(50.6 \%)$

$0.180^{b}$

152 (52.8\%)

18 (6.3\%)

270 (93.7\%)

55 (5.3\%)

$0.402^{b}$

981 (94.7\%)

502 (48.5\%)

$0.022^{b}$

$165(57.3 \%)$

534 (51.5\%) 
Table 3 Gross and adjusted multilevel logistic regression analysis of factors associated with overweight in children of municipal public schools in Northern Brazil, 2018

\begin{tabular}{|c|c|c|c|c|}
\hline \multirow[t]{2}{*}{ Variables } & \multicolumn{2}{|c|}{ Gross Analysis } & \multicolumn{2}{|c|}{ Adjusted Analysis } \\
\hline & $\overline{\mathrm{OR}}(95 \% \mathrm{Cl})$ & $\begin{array}{l}\text { Model I } \\
\text { OR }(95 \% \mathrm{Cl})\end{array}$ & $\begin{array}{l}\text { Model II } \\
\text { OR }(95 \% \mathrm{Cl})\end{array}$ & $\begin{array}{l}\text { Model III } \\
\text { OR (95\%Cl) }\end{array}$ \\
\hline
\end{tabular}

\section{Specific Individual Average Effects}

\section{Socio-demographic characteristics}

Gender

Female

Male

Age

Grade

Second

Fourth

\section{Physical aptitude}

Distance covered in $6 \mathrm{~min} /$ height $(\mathrm{m})$

Non-sedentary activities classification

$<2$ activities

$\geq 2$ activities

Sedentary activities classification

$<2$ activities

$\geq 2$ activities

Food Consumption Adequacy

Meals

Insufficient ( $<5$ meals)

Adequate ( $>5$ meals)

Cereals group

Insufficient ( $<6$ portions)

Adequate ( $\geq 6$ portions)

Vegetables group

Insufficient ( $<3$ portions)

Adequate ( $>3$ portions)

Fruits group

Insufficient ( $<3$ portions)

Adequate ( $\geq 3$ portions)

Meats and eggs group

Insufficient ( $<1$ portion)

Adequate (1-2 portions)

Excessive ( $>2$ portions)

Dairy group

Insufficient ( $<3$ portions)

Adequate (3 portions)

Legume group

Insufficient ( $<1$ portion)

Adequate (1-2 portions)

Excessive (> 2 portions)

Fat group
1

$0.89(0.69-1.17)$

$0.996(0.88-1.12)$

1

$1.08(0.83-1.42)$

$0.996(0.99-1.00)^{*}$

1

$0.83(0.63-1.10)$

1

$1.49(1.13-1.98)^{*}$

$1.48(0.22-0.43)^{*}$

$1.46(1.09-1.95)^{*}$

$0.88(0.67-1.16)$

$0.78(0.57-1.05)$

$0.80(0.59-1.08)$
1

$0.73(0.38-1.41)$

1

$1.04(0.55-1.97)$

1

$1.21(0.80-1.81)$

1

$1.43(0.94-2.16)$

$1.30(0.79-2.15)$

1

$1.18(0.67-2.09)$

1

$0.90(0.65-1.23)$

$0.66(0.33-1.32)$ 
Table 3 Gross and adjusted multilevel logistic regression analysis of factors associated with overweight in children of municipal public schools in Northern Brazil, 2018 (Continued)

\begin{tabular}{|c|c|c|c|c|}
\hline \multirow[t]{2}{*}{ Variables } & \multicolumn{2}{|l|}{ Gross Analysis } & \multicolumn{2}{|l|}{ Adjusted Analysis } \\
\hline & OR $(95 \% \mathrm{Cl})$ & $\begin{array}{l}\text { Model I } \\
\text { OR (95\%Cl) }\end{array}$ & $\begin{array}{l}\text { Model II } \\
\text { OR }(95 \% \mathrm{Cl})\end{array}$ & $\begin{array}{l}\text { Model III } \\
\text { OR }(95 \% \mathrm{Cl})\end{array}$ \\
\hline Adequate ( $\leq 1$ portion) & 1 & & & \\
\hline Excessive (> 1 portions) & $1.24(0.91-1.69)$ & & & \\
\hline \multicolumn{5}{|l|}{ Sugar group } \\
\hline Adequate ( $\leq 1$ portion) & 1 & & 1 & 1 \\
\hline Excessive (> 1 portions) & $1.35(1.02-1.78)^{*}$ & & $1.36(1.00-1.84)^{*}$ & $1.26(0.93-1.70)$ \\
\hline \multicolumn{5}{|c|}{ Specific Contextual Average Effects } \\
\hline \multicolumn{5}{|l|}{ School's characteristics } \\
\hline \multicolumn{5}{|l|}{ Administrative region } \\
\hline North & 1 & & & 1 \\
\hline Center-South & $0.72(0.52-1.02)$ & & & $0.82(0.51-1.31)$ \\
\hline South & $0.47(0.34-0.66)^{*}$ & & & $0.52(0.33-0.83)^{*}$ \\
\hline \multicolumn{5}{|l|}{ School shift } \\
\hline Part-time & 1 & & & \\
\hline Full-time & $0.64(0.49-0.84)^{*}$ & & & \\
\hline \multicolumn{5}{|c|}{ Physical activity practice at school } \\
\hline \multicolumn{5}{|l|}{ Dance } \\
\hline No & 1 & & & 1 \\
\hline Yes & $0.63(0.48-0.83)^{*}$ & & & $0.63(0.43-0.92)^{*}$ \\
\hline \multicolumn{5}{|l|}{ Body practices } \\
\hline No & 1 & & & \\
\hline Yes & $0.69(0.52-0.90)^{*}$ & & & \\
\hline \multicolumn{5}{|c|}{ Weekly physical activity classes } \\
\hline$<3$ weekly classes & 1 & & & \\
\hline$\geq 3$ weekly classes & $0.64(0.49-0.84)^{*}$ & & & \\
\hline
\end{tabular}

\section{School feeding}

Number of offered meals

1 meal

1

3 meals

$0.62(0.47-0.81)^{*}$

The school has a garden

No

1

Yes

$0.73(0.56-0.96)^{*}$

\section{Foods Sale in School Surroundings}

Food sale in school surroundings

No

Yes

Fried snacks sale

No

Yes

Candy sale

No

Yes

Sweetened beverage sale
$1.45(0.97-2.17)$

1

$1.38(1.05-1.81)^{*}$

1

$1.58(1.11-2.27)^{*}$
1

$1.67(1.01-2.75)^{*}$ 
Table 3 Gross and adjusted multilevel logistic regression analysis of factors associated with overweight in children of municipal public schools in Northern Brazil, 2018 (Continued)

\begin{tabular}{|c|c|c|c|c|}
\hline \multirow[t]{2}{*}{ Variables } & \multicolumn{2}{|l|}{ Gross Analysis } & \multicolumn{2}{|l|}{ Adjusted Analysis } \\
\hline & $\overline{O R}(95 \% \mathrm{Cl})$ & $\begin{array}{l}\text { Model I } \\
\text { OR (95\%Cl) }\end{array}$ & $\begin{array}{l}\text { Model II } \\
\text { OR }(95 \% \mathrm{Cl})\end{array}$ & $\begin{array}{l}\text { Model III } \\
\text { OR }(95 \% \mathrm{Cl})\end{array}$ \\
\hline No & 1 & & & \\
\hline Yes & $0.77(0.59-1.01)$ & & & \\
\hline \multicolumn{5}{|c|}{ SHP actions performed at school } \\
\hline \multicolumn{5}{|c|}{ Healthy eating promotion activities } \\
\hline No & 1 & & & \\
\hline Yes & $1.72(0.79-3.75)$ & & & \\
\hline \multicolumn{5}{|c|}{ Nutritional state assessment in the school } \\
\hline No & 1 & & & \\
\hline Yes & $0.66(0.45-0.96)^{*}$ & & & \\
\hline \multicolumn{5}{|c|}{ Childhood obesity prevention activities } \\
\hline No & 1 & & & \\
\hline Yes & $0.42(0.16-1.01)$ & & & \\
\hline \multicolumn{5}{|c|}{ Nutritional state assessment in the PHC } \\
\hline No & 1 & & & \\
\hline Yes & $1.46(1.10-1.95)^{*}$ & & & \\
\hline \multicolumn{5}{|c|}{ General Contextual Effects - Measuring the variation between the nutritional status classification } \\
\hline$\sigma^{2}(\mathrm{SE})$ & & 0.411 & $0.386(0.110)$ & $0.102(0.161)$ \\
\hline PCV & & $(0.114)$ & $2.36 \%$ & $23.68 \%$ \\
\hline ICC & & & $4.32 \%$ & $0.03 \%$ \\
\hline MOR & & $4.88 \%$ & 1.44 & 1.10 \\
\hline AUC & & 1.48 & $0.652(0.615-0.690)$ & $0.637(0.599-0.675)$ \\
\hline AUC change* & & $1.0(1.0-1.0)$ & -0.348 & -0.015 \\
\hline \multicolumn{5}{|c|}{ Model evaluation } \\
\hline Log likelihood & & -606.44045 & -600.10343 & -589.59713 \\
\hline LR Test & & & 0.0054 & 0.0003 \\
\hline AIC & & 1216.881 & 1210.207 & 1197.194 \\
\hline
\end{tabular}

Note: ${ }^{*} \mathrm{p}<0.05$, PHC Primare Health Care, $\sigma^{2}$ Contextual level variance, SE Standard Error, PCV Proportional Change of Variance, ICC Interclass Correlation Coefficient, MOR Odds Ratio median, AUC Area under the receiver operating characteristic curve, $L R$ test Likelihood ratio test, AIC Akaike Information Criterion. * Change in relation to the previous model

obesity were lower in children who studied in Southern Palmas and had dance classes, but higher in those who were in the Center-South and who could buy candies in the school surroundings. The inclusion of these variables did not cause major changes in the magnitude of the association of overweight/obesity with individual variables; only food portions belonging to the sugar group lost significance.

From model I (null model) to model II (level 1), we verified a reduction in variance from 0.411 to 0.386 after the inclusion of individual variables. In model II, with the inclusion of individual variables, we found a reduction in ICC and MOR from model I to model II, being 4.88 to $4.32 \%$ and 48 to $44 \%$, respectively. The PCV explained only $2.36 \%$, which demonstrates that the individual variables explain a small part of the variation (Table 3). The AUC curve showed a value of 0.652 with -0.348 change (Fig. 1), showing a low discriminatory prediction, thus individual level variables are insufficient to distinguish overweight/obesity.

The inclusion of the contextual variables in model III demonstrated that the variance of overweight/obesity between model II (0.386) and model III (0.102) reduced, explaining $23.7 \%$ of the variation, thus contextual variables of the school environment improve the explanation of the variation in overweight/obesity. From model II to model III, we observed a considerable decrease in the conditional ICC to $0.03 \%$ and MOR to 1.10 , that is, the GCE is well explained by the SCE of the school environment (Table 3), and -0.015 reduction of the AUC curve 


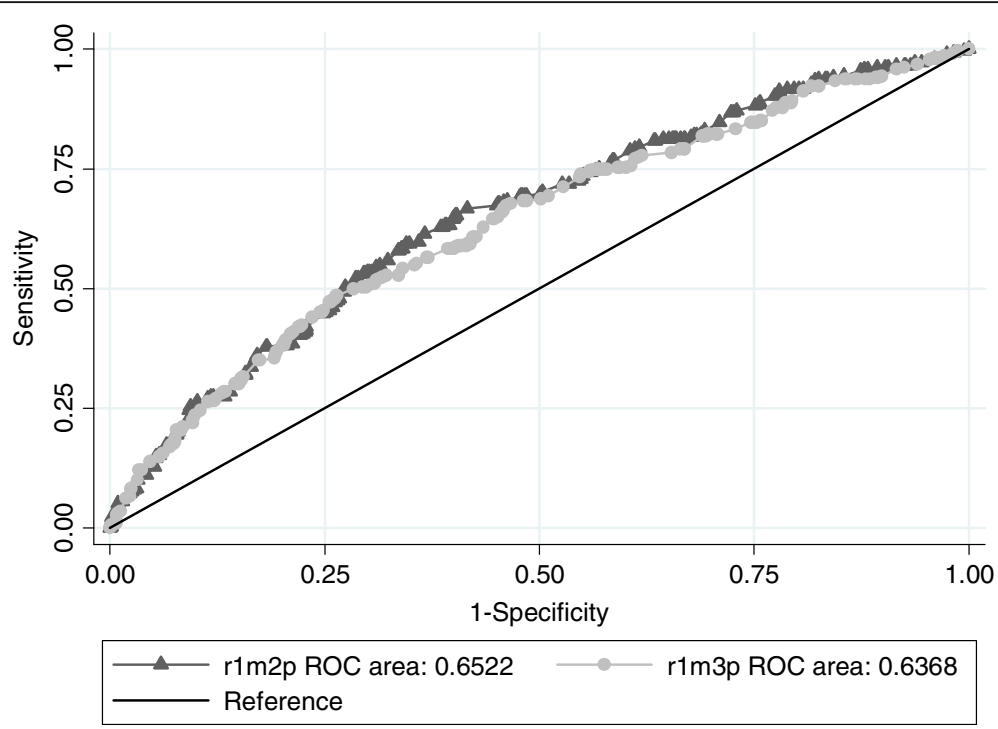

Fig. 1 AUC curve of Model II and Model III of adjusted multilevel logistic regression analysis of factors associated with overweight in children of municipal public schools in Northern Brazil, 2018

(Fig. 1). We identified the reduction of AIC between models II and III and the likelihood ratio test indicated better adequacy of model III.

\section{Discussion}

We identified a high prevalence of overweight/obesity in children who studied at schools enrolled with the SHP. These values were higher than those found in Latin America [11, 42], but lower than those of children in the Brazilian urban environment [43]. Comparing with researches in other regions of Brazil, these values were lower than those reported for the South [44-47], Southeast [48-50], and Northeast [51, 52], but higher than those from Central-West [53-55], Southeast [56-58], and Northeast [59-61].

We found that being overweight/obesity was associated with high consumption of sugar-rich foods, performance of $2+$ sedentary activities/day, and cardiorespiratory fitness reduction, results that were similar to those reported elsewhere [2]. In relation to high intake of foods rich in sugar, our study evaluated sugary foods and sweetened drinks containing large amount of simple carbohydrates with high energy density. These foods have been shown to be associated with obesity and diabetes positive due to positive energy balance and consequent, weight gain and body fat [62]. This confirms the importance of investing in intervention strategies and projects aimed at decreasing the consumption of candies and sweetened beverages, increasing the consumption of fruits and vegetables, and reducing fats and eating out habits [63].

Regarding sedentary activities, these were associated with reduced physical activity [2] development of cardiometabolic risk factors [64], NCDs and premature mortality [65], and quality of consumed food, such as higher candies intake $[2,64,66]$. This can be explained by the fact that sedentary activities reduce energy expenditure and tend to increase the chance of consuming unhealthy foods, thus creating a positive energy balance and consequently weight gain [2].

It is also verified that screen time on TV and computers determines children's exposure to food advertisements which can influence food intake and preference. Food intake while watching TV and playing on the computer distracts children, promoting unintentional excessive eating [67]. According to Lipsky [66], foods mostly consumed during this period are sugary drinks, fast food, refined grains and calorie dense foods, and low amounts of fruits and vegetables. The negative association found for the cardiorespiratory fitness test can be due to (i) physical activity represents $20-40 \%$ of total energy expenditure, (ii) that $60 \mathrm{~min}$ of this practice contribute to weight control, and (iii) $150 \mathrm{~min}$ of moderate activity/ week reduce blood pressure $[68,69]$ and visceral fat $[63$, 68], improve lipid profile and insulin sensitivity [63], and increase life expectancy by 0.68 years [65].

We found an association between overweight/obesity and school-related variables such as school shift and location, amounts of physical activity and offered meals, cafeteria conditions, presence of school gardens and other types of grown food, food sale in the school surroundings and its quality, and existence of nutritional diagnosis.

Studies have demonstrated that school location is related to the environmental causes of obesity, which impact food access and consumption and physical activity [70-72], depending on the availability of leisure spaces 
and food selling sites [73, 74]. Extended school shifts strengthen socialization and cultural diffusion [72, 75], contributing to increased body practices [70, 72, 75], promotion of healthy eating by having $30-50 \%$ of meals at schools [2, 70, 72, 76], increased consumption of natural/minimally processed foods, and reduced consumption of their ultra-processed counterparts [72].

Another important finding of this study relates to the fact that the greater the offer of body practices at school, the lower the prevalence of overweight/obesity, which was herein attributed to institutions that work full time and offer dance classes. A Brazilian study in public and private schools had a similar result [61], which meets the fact that physical activity can be reinforced through the scholar curriculum [9-13, 63, 77-79]. Nonetheless, it is necessary for schools to hire physical education professionals to guide activities and adapt physical structures, often assessed as inadequate [80].

We also highlight the role played by the number of meals at school, e.g., children should have at least three meals at school, where they stay for $5-7 \mathrm{~h}$, making school feeding programs $[2,63,70,76,81]$ such as the National School Feeding Program (NSFP) [82] relevant for Brazilian public schools as these offer quality food and stimulate healthy food choices [70, 76], such as fruits and vegetables $[63,80]$. As for the cafeterias, this association can be explained by the influence of the atmosphere within an appropriate environment for food consumption [76].

Another relevant factor regarding school feeding was the presence of gardens that provided fruits and vegetables, cereals, tubers, and spices for school feeding, as well as the existence of a pedagogical space for foodrelated and nutritional education practices, allowing better knowledge on nutrition, food preparation, and healthy eating habits [83-87]. We did not, however, find this association in our study.

As for food sale, we observed similar results in studies that assessed the relationship between childhood obesity and the environment in which children live, emphasizing that shorter distances to and higher occurrences of places selling ultra-processed food are associated with more monotonous food choices with high caloric density, and, as consequence, with higher prevalence of overweight/obesity [73, 74, 88]. In Brazil's case, although it is positive that NSFP [82] does not allow food sale in public schools, it is still necessary to propose legal provisions that control food trade in school surroundings [81], including the informal ones.

The scientific literature also points out that performing nutritional diagnosis at schools was also associated with being overweight/obesity $[68,69]$, i.e., this is an essential instrument for assessing nutritional status [9-13, 77-79], allowing better management of overweight/ obesity by PHC for individual and/or collective care [68, 69]. Because children spend most of their daytime at school, it denotes a relevant social equipment for the diagnosis and monitoring of overweight/obesity, with training and standardization of this process being required for the proper, efficient care of the population [68].

It is noteworthy that, in our study, food and nutrition education interventions were not associated, having as potential reasons the low frequency of these actions [68, $69,84,89-91]$ and the poor quality of both approach and content [84, 89, 91, 92]. Taveras et al. [92] demonstrated that behavioral interventions helped to improve BMI/A and motivate habit changes in children with more frequency in the activities. Studies have shown the role of schools as promoters of health and permanent healthy habits $[63,69,84,85$, 90], highlighting the relevance of intersectoral actions with the engagement of teachers and health care professionals [9-13, 77-79].

There are no studies conducted in Brazil evaluating the association between overweight/obesity and individual and school variables, whereas international studies $[93,94]$ do not allow comparison as these were carried out in private and public schools, and the latter did not provide free food for all children as in Brazil [82]. From the aforementioned individual variables, we found in model II that performing $2+$ sedentary activities/day and having five meals/day, besides consuming $1+$ portions of candy/day, were related to being overweight/obesity. In carrying out model III, the inclusion of contextual analyses considered the permanence of sedentary activities, number of meals, school location, school shift, dance classes, and candy sale in the school surroundings. We observed a reduction in variance in the models, showing that the individual and contextual variables together improve the explanation of the prevalence of overweight/obesity.

In the study by Fox et al. [93], a similarity of the relationship between overweight/obesity and candy purchase at or around the school could be observed, but it did not show any association between environment and eating habits at school and BMI/A. Li et al. [94], when evaluating the relationship among overweight/obesity and individual, family, school, and community variables, observed that children with higher participation in sports had lower BMI/A and positive association with longer sedentary activities, data that corroborate our study.

Regarding variation by ICC, the schools presented a small variation, but with a good reduction when the contextual variables of the school environment are included, however the AUC curve shows that the individual and contextual variables have low, but similar, predictive values. Regarding the measure of heterogeneity by MOR, we found that school explained almost half of the child's 
chance of being overweight/obese, showing an improvement after the inclusion of contextual variables.

When evaluating the GCE for heterogeneity and variation, we found that we may not have considered other contextual and individual variables that improve prediction. Also, contexts such as the neighborhood and family may be involved in the explanation of overweight/obesity. Thus, it is relevant that new studies consider these factors. It should be added that the reduced values may be related to the fact that the school may present heterogeneity of the enrolled children, that is, some children may not live so close to the schools, which would not be related to geographic evaluation. For this reason, we used specific variables of the school environment to better understand the variation of the explanation of the school in the development of childhood obesity.

However, we reinforce the relevance of the school in the control of childhood obesity and in the promotion of strategic actions for the promotion of healthy eating habits and physical activity. Studies show that school is a space to prevent and reduce childhood obesity since it influences healthy eating, weight control and maintenance, and health in general. Furthermore, children consume up to $50 \%$ of their daily calories at school $[95,96]$, which is a positive point for Brazil since NSFP for a menu composed of quality food [82] and in adequate quantity, substitution of high calorie dense food; presence of school gardens improving the supply of fruits and vegetables [96] and their preference among children; and the encouragement of physical activity [95, 96], in addition to health education programs [95]. The implementation of the programs and policies presents many challenges related to the availability of trained professionals, organization of schools for the implementation and evaluation and monitoring of programs and policies $[95,96]$.

As a limitation of the study, it is emphasized that food consumption and daily activity practices may be under or overestimated; however, as a control, we used an agevalidated instrument developed by nutritionists, physical education teachers, psychologists, and educators, in addition to children that were trained to fill the questionnaire and activity follow up [28, 34].

Sedentary and non-sedentary activities were estimated with self-reported data which can present inaccuracies, which is why we chose to use a validated instrument. For future studies, the use of questionnaires that do not aim solely for an estimate is recommended. Although the 6-min walk test was validated, differences in values can be linked to ethnicity [24, 27], culture [27], socioeconomic factors, climate [97], methodological variations during the test $[24,27]$, and motivation during walk [23]. However, we emphasize that our study followed the guidelines of the American Thoracic Society [22] to ensure standard measurements.

\section{Conclusion}

Overweight/obesity was associated with individual and school environment variables, highlighting important implications of schools in the implementation of PHC policies and programs, with an environmental and behavioral approach. Thus, the needs to implement and supervise regulatory measures for food sale in the school surroundings as well as to increase the availability of full-time schools that allow children to eat more meals and to participate in activities that stimulate body practices are emphasized.

In this context, we highlight the importance of intersectoral activities, especially education and health, with emphasis on food-related and nutritional education and physical activity, which should be included in the scholar curriculum. Thus, ongoing training and education of health and school professionals, aiming at more effective and sustainable actions to control childhood obesity, must also be on the agenda of local and national governments.

\section{Abbreviations \\ $\sigma^{2}$ : Contextual level variance; 95\%Cl: 95\% confidence interval; ACVR: Absence of cardiovascular risk; AIC: Akaike information criterion; AUC: Area under the receiver operating characteristic; BMI/A: Body mass index for age; NCDs: Chronic non-communicable diseases; T6M: Distance/height; GCE: General contextual effects model; T6M/t: Index walked distance/height, both in meters; ICC: Interclass Correlation Coefficient; LR test: Likelihood ratio test; MOR : Median odds ratio; NSFP: National School Feeding Program; OR: Odds ratio; PCVR: Presence of cardiovascular risk; PHC: Primary health care; PCV: Proportional change of variance; SHP: School Health Program; SCE: Specific contextual effects model; SE: Standard error; WC: Waist circumference; WHtR: Waist height to ratio; WHO: World Health Organization}

\section{Acknowledgements}

The authors thank the Ministry of Education (Coordenação de

Aperfeiçoamento de Pessoal de Nível Superior - CAPES) for funding the project [Publication 023/2014 CAPES/DINTER Inter-institutional Graduate Scholarship Program], the Department of Nutrition and Health at Universidade Federal de Viçosa, and the team of researchers with the School of Nutrition at Universidade Federal de Tocantins for their contribution in data collection and consolidation. Directorship, pedagogical, and administrative teams of the municipal public schools as well as the volunteers who participated in the research are also gratefully acknowledged.

\section{Authors' contributions}

RAMM, TRM and RMMC conceived conceptualization; RAMM, TRM, GDC, LCVC and RMMC conceived methodology and approved the final manuscript; RAMM and TRM realized statistical analysis; RAMM and RMMC secured funding, were responsible to project administration and supervision; RAMM performed the data collection and data curation, and wrote original draft; TRM, GDC, LCVC and RMMC wrote review and editing draft.

\section{Funding}

This work was supported by the Coordination for the Improvement of Higher Education Personnel (Coordenação de Aperfeiçoamento de Pessoal de Nivel Superior - CAPES) of the Ministry of Education [Publication 023/2014 CAPES/DINTER Inter-institutional Graduate Scholarship Program]. The role of the funding agency was only to provide materials for data collection.

\section{Availability of data and materials}

The acquired and/or analyzed data are not publicly available because of the policies of the Palmas Municipal Departments of Education and Health, the lack of authorization from the children's legal guardians, and the agreement with the Research Ethics Committee that the database would remain with 
the corresponding author only. However, all data can be made available by the corresponding author upon reasonable request.

\section{Ethics approval and consent to participate}

The project was approved by the Palmas Municipal Departments of Education and Health and by the Research Ethics Committee of the Universidade Federal de Viçosa ( $n^{\circ}$ 2.733.549). Written agreement and consent were obtained from legal guardians and minors, respectively.

\section{Consent for publication}

Not Applicable.

\section{Competing interests}

The authors declare that they have no competing interests.

\section{Author details}

${ }^{1}$ Curso de Nutrição, Câmpus de Palmas, Universidade Federal do Tocantins, Quadra 109 Norte, Avenida NS 15, ALCNO-14, Bloco de Apoio Logístico e Administrativo 1 (BALA1) $2^{\circ}$ andar, sala 19, Curso de Nutrição. Bairro, Plano Diretor Norte, Palmas, Tocantins 77001-090, Brazil. ${ }^{2}$ Departamento de Medicina e Enfermagem, Universidade Federal de Viçosa, Avenida PH Rolfs, s/n, Câmpus Universitário, Bairro Centro, Viçosa, Minas Gerais 36570-900, Brazil. ${ }^{3}$ Departamento de Nutrição e Saúde, Universidade Federal de Viçosa, Avenida PH Rolfs, s/n, Prédio CCBII, Câmpus Universitário, Bairro Centro, Viçosa, Minas Gerais 36570-900, Brazil.

\section{Received: 21 January 2020 Accepted: 20 April 2020}

Published online: 28 April 2020

\section{References}

1. World Health Organization. World health statistics 2018: monitoring health for the SDGs, sustainable development goals. Geneva: WHO; 2018. Available at https://apps.who.int/iris/bitstream/handle/10665/272596/9789241565585eng.pdf?ua=1. Acessed 13 August 2019.

2. Kumar S, Kelly AS. Review of childhood obesity: from epidemiology, etiology, and comorbidities to clinical assessment and treatment. Mayo Clin Proc. 2017;92(2):251-65. https://doi.org/10.1016/j.mayocp.2016.09.017.

3. Cano GS, Ciges RB, Sanchez-Martinez B, et al. Overweight and childhood obesity according to socioeconomic variables in third grade school-age children in the city of Barcelona. Nutr Hosp. 2019;36(5):1043-8. https://doi. org/10.20960/nh.02205.

4. Swinburn BA, Kraak VI, Allender S, et al. The global syndemic of obesity, undernutrition and climate change: the lancet commission report. Lancet Comissions. 2019;393(10173):791-846. https://doi.org/10.1016/S01406736(18)32822-8.

5. Story $M$, Kaphingst KM, O'Brien RR, et al. Creating healthy food and eating environments: policy and environmental approaches. Annu Rev Public Health. 2008;29:253-72. https://doi.org/10.1146/annurev.publhealth.29. 020907.090926 .

6. Roberto CA, Swinburn B, Hawkes C, et al. Patchy progress on obesity prevention: emerging examples, entrenched barriers, and new thinking. Lancet. 2015;385(9985):2400-9. https://doi.org/10.1016/S01406736(14)61744-X.

7. Brasil. Lei $n^{\circ} 11.947,16$ de julho de 2009; 2009. Available at http://www. planalto.gov.br/ccivil_03/_Ato2007-2010/2009/Lei/L11947.htm. Acessed 25 Jan 2019.

8. Brasil. Ministério da Saúde. Departamento de Atenção Básica. Política Nacional de Atenção Básica. Brasília: Ministério da Saúde; 2012. Available at http://189.28.128.100/dab/docs/publicacoes/geral/pnab.pdf. . Acessed 10 Jan 2019.

9. Organización Mundial de la Salud. Estabelecimiento de áreas de acción prioritarias Para la prevención de la obesidade infantil: conjunto de herramientas Para que los estados miembros determinen e identifiquen áreas de acción prioritarias. Geneva: WHO; 2016. Available at https://apps. who.int/iris/bitstream/handle/10665/250750/9789243503271-spa. pdf?sequence=1. Acessed 03 Mar 2018.

10. World Health Organization. Report of the Comission on ending childhood obesity. Geneva: WHO; 2016. Available at https://apps.who.int/iris/bitstream/ handle/10665/204176/9789241510066_eng.pdf?sequence=1. Acessed 03 Mar 2018.
11. Organização Pan-Americana de Saúde, Organização Mundial de Saúde. Plano de ação Para prevenção da obesidade em crianças e adolescentes. Washington: OPAS; 2014. Available at https://www.paho.org/bra/images/ stories/UTFGCV/planofactionchildobesity-por.pdf?ua=1. Acessed 03 Mar 2018.

12. Brasil. Decreto $n^{\circ} 6.286$ de 5 de dezembro de 2007. Institui o Programa Saíde na Escola e dá outras providências; 2007. Available at http:// wwwplanaltogovbr/ccivil_03/_Ato2007-2010/2007/Decreto/D6286htm Acessed 05 Apr 2018.

13. Brasil. Portaria Interministerial $n^{\circ} 1.055$ de 25 de abril de 2017; 2017. Available at http://bvsms.saude.gov.br/bvs/saudelegis/gm/2017/pri1055_26_ 04_2017.html. Acessed 05 Apr 2018.

14. Instituto Brasileiro de Geografia e Estatística. Cidades - Palmas, Tocantins; 2017. Available at http://cidades.ibge.gov.br/xtras/perfil.php?lang= \&codmun=520870\&search=\|inf ogr\%E1 ficos:-informa\%E7\%F5es-completas. Acessed 05 Apr 2019.

15. Palmas. Escolas Municipais; 2017. Available at https://www.palmas.to.gov.br/ servicos/escolas-municipais/56/. Acessed 18 Sep 2017.

16. Instituto Nacional de Estudos e Pesquisas Educacionais Anísio Teixeira Sinopse Estatística de Educação Básica 2016. Brasília: INEP; 2017. http:// portal.inep.gov.br/sinopses-estatisticas-da-educacao-basica. Acessed 07 Apr 2019.

17. Brasil. Orientações Para a coleta e análise de dados antropométricos em serviços de saúde: Norma Técnica do Sistema de Vigilância Alimentar e Nutricional - SISVAN. Brasília: Ministério da Saúde; 2011. Available at http:// bvsms.saude.gov.br/bvs/publicacoes/orientacoes_coleta_analise_dados_ antropometricos.pdf. Acessed 18 Sep 2017.

18. Brasil. Incorporação das curvas de crescimento da Organização Mundial da Saúde de 2006 e 2007 no Sistema de Vigilância Alimentar e Nutricional SISVAN. Brasília: Ministério da Saúde; 2007. Available at https://www.sprs. com.br/sprs2013/bancoimg/131209104419oms2006_2007.pdf Acessed 18 Sep 2017.

19. World Health Organization. WHO AntroPlus for personal computers: software for assessing growth of the world's children and adolescents. Geneva: WHO; 2009.

20. Frisancho AR. Anthropometric standards for the assessment of growth and nutritional status. 2nd ed. Michigan: University of Michigan Press; 2008.

21. Ashwell M, Gibson S. A proposal for a primary screening tool: 'keep your waist circumference to less than half your height'. BMC Med. 2014;12(207): 2-6. https://doi.org/10.1186/s12916-014-0207-1.

22. American Thoracic Society. ATS statement: guidelines for the six-minute walk test. Am J Respir Crit Care Med. 2002;166(1):111-7. https://doi.org/10. 1164/ajrccm.166.1.at110.

23. Kain J, Leyton B, Concha F, et al. Evaluación de una intervención en educación alimentaria y actividad física Para prevenir obesidad infantil en escuelas públicas de Santiago de Chile. Arch Latinoam Nutr. 2012;62(1):60-7 URL: http://ve.scielo.org/scielo.php?script=sci_arttext\&pid=S0004-06222012 000100009\&lng=es\&nrm=iso.

24. Geiger R, Willeit J, Rummel M, et al. Six-minute walk distance in overweight children and adolescents: effects of a weight-reducing program. J Pediatr. 2011;158(3):447-51. https://doi.org/10.1016/j.jpeds.2010.08.020.

25. Druce Axley J, Werk LN. Relationship between abdominal adiposity and exercise tolerance in children with obesity. Pediatr Phys Ther. 2016;28(4): 386-91. https://doi.org/10.1097/pep.0000000000000284.

26. Mestre MN, Audag N, Caty G, et al. Learning and encouragement effects on six-minute walking test in children. J Pediatr. 2018;198:98-103. https://doi. org/10.1016/j.jpeds.2018.02.073.

27. Mylius CF, Paap D, Takken T. Reference value for the 6-minute walk test in children and adolescents: a systematic review. Expert Rev Respir Med. 2016; 10(12):1335-52. https://doi.org/10.1080/17476348.2016.1258305.

28. Universidade Federal de Santa Catarina. Sistema de Monitoramento de Consumo Alimentar e Atividade Física de Escolares - manual de Diretores (as). Santa Catarina: UFSC; 2013. Available at http://caafe.ufsc.br/public/ uploads_midias/1376617230.pdf. Acessed 10 Apr 2017

29. Assis MAA, Guimarães D, Calvo MCM, et al. Reprodutibilidade e validade de questionário de consumo alimentar Para escolares. Rev Saúde Pública. 2007; 41(6):1054-7. https://doi.org/10.1590/S0034-89102007000600022.

30. Assis MAA, Benedet J, Kerpel $R$, et al. Validação da terceira versão do Questionário Alimentar do Dia anterior (QUADA-3) Para escolares de 6 a 11 anos. Cad Saude Publica. 2009;25(8):1816-26. https://doi.org/10.1590/S0102$311 \times 2009000800018$. 
31. Davies VF, Kupek E, Assis MSS, et al. Validation of a web-based questionnaire to assess the dietary intake of Brazilian children aged 7-10 years. J Hum Nutr Diet. 2015;28(Suppl1):93-102. https://doi.org/10.1111/jhn. 12262.

32. Barros MVG, Assis MAA, Pires MC, Grossemann S, et al. Validity of physical activity and food consumption questionnaire for children aged seven to ten years old. Revista Brasileira de Saúde Materno Infantil. 2007;7(4):43-448. https://doi.org/10.1590/S1519-38292007000400011.

33. Lobo AS, Assis MAA, Barros MVG, et al. Reprodutibilidade de um questionário de consumo alimentar Para crianças em idade escolar. Revista Brasileira de Saúde Materno Infantil. 2008;8(1):55-63. https://doi.org/10.1590/ S1519-38292008000100007

34. Costa FF, Schmoelz CP, Davies VF, et al. Assessment of diet and physical activity of Brazilian schoolchildren: usability testing of a web-based questionnaire. JMIR Res Protoc. 2013;2(2):e31. https://doi.org/10.2196/ resprot.2646.

35. Brasil. Ministério da Saúde. Departamento de Atenção Básica. Guia alimentar Para a população Brasileira: promovendo a alimentação saudável. Brasília: Ministério da Saúde; 2008. Available at http://bvsms.saude.gov.br/bvs/ publicacoes/guia_alimentar_populacao_brasileira_2008.pdf Acessed 10 Apr 2017.

36. Merlo J, Wagner P, Ghith $N$, et al. An original stepwise multilevel logistic regression analysis of discriminatory accuracy: the case of neighbourhoods and health. PLoS One. 2016;11(4):e0153778. https://doi.org/10.1371/journal. pone.0153778.

37. Merlo J, Wagnera P, Austind PC, et al. General and specific contextual effects in multilevel regression analyses and their paradoxical relationship: a conceptual tutorial. SSM - Population Health. 2018;5(2018):33-7. https://doi. org/10.1016/j.ssmph.2018.05.006.

38. Austin PC, Merlo J. Intermediate and advanced topics in multilevel logistic regression analysis. Stat Med. 2017;36:3257-77. https://doi.org/10.1002/sim. 7336.

39. Merlo J, Chaix B, Ohlsson $\mathrm{H}$, et al. A brief conceptual tutorial of multilevel analysis in social epidemiology: using measures of clustering in multilevel logistic regression to investigate contextual phenomena. J Epidemiol Community Health. 2006;60(4):290-7. https://doi.org/10.1136/jech.2004. 029454

40. Larsen K, Petersen JH, Budtz-Jorgensen E, et al. Interpreting parameters in the logistic regression model with random effects. Biometrics. 2000;56(3): 909-14. https://doi.org/10.1111/j.0006-341x.2000.00909.x.

41. Larsen K, Merlo J. Appropriate assessment of neighborhood effects on individual health: integrating random and fixed effects in multilevel logistic regression. Am J Epidemiol. 2005;161(1):81-8. https://doi.org/10.1093/aje/ kwi017.

42. Rivera JA, Cossío TG, Pedraza LS, et al. Childhood and adolescent overweight and obesity in Latin America: a systematic review. Lancet Diabetes Endocrinol. 2014;2(4):321-32. https://doi.org/10.1016/S22138587(13)70173-6.

43. Instituto Brasileiro de Geografia e Estatística. Pesquisa de orçamentos familiares 2008-2009: Antropometria e estado nutricional de crianças, adolescentes e adultos no Brasil. Rio de Janeiro: IBGE; 2010. Available at https://bibliotecaibgegovbr/visualizacao/livros/liv45419pdf Acessed 15 May 2017.

44. Malinski MP, Voser RC. Sobrepeso e obesidade em jovens escolares. Arquivos de Ciências da Saúde. 2016;23(1):68-72. https://doi.org/10.17696/ 2318-3691.23.1.2016.49

45. Souza M, Spessato B, Coutinho M, et al. Estado nutricional, idade e sexo influenciam os níveis de atividade física de escolares? Revista Brasileira de Atividade Física \& Saúde. 2015;20(6):598-607. https://doi.org/10.12820/rbafs. v.20n6p598.

46. Souza MPS, Benneman RM. Antropometria e estado nutricional de escolares adolescentes do ensino fundamental da rede municipal de ensino da cidade de Maringá-PR no ano de 2011. In: CESUMAR, editor. VIII EPCC: Encontro Internacional de Produção Científica Cesumar. Maringá: Editora CESUMAR; 2011. Available at http://www.cesumar.br/prppge/pesquisa/ epcc2013/oit_mostra/Marizete_Pereira_dos_Santos_Souza.pdf Acessed 10 Sep 2019.

47. Bernardo CO, Pudla KJ, Longo GZ, et al. Fatores associados ao estado nutricional de escolares de 7 a 10 anos: aspectos sociodemográficos, de consumo alimentar e estado nutricional dos pais. Rev Bras Epidemiol. 2012; 15(3):651-61. https://doi.org/10.1590/S1415-790X2012000300018.
48. Miranda JMQ, Palmeira MV, Polito LFT, et al. Prevalência de sobrepeso e obesidade infantil em instituições de ensino: públicas vs. privadas. Rev Bras Med Esporte. 2015;21(2):104-7. https://doi.org/10.1590/1517869220152102143660

49. Costa RF, Cintra IP, Fisberg M. Prevalência de sobrepeso e obesidade em escolares da cidade de Santos, SP. Arq Bras Endocrinol Metabol. 2006;50(1): 60-7. https://doi.org/10.1590/S0004-27302006000100009.

50. Pardo IM, Mercadante MP, Zanatta MF, et al. Prevalência de excesso de peso entre estudantes de ensino fundamental de escola pública e privada em Sorocaba, São Paulo, Brasil. Revista Brasileira de Medicina da Família e Comunidade. 2013;8(26):43-50. https://doi.org/10.5712/rbmfc8(26)545.

51. Albuquerque LP, Cavalvante ACM, Almeida PC, et al. Relação da obesidade com o comportamento alimentar e o estilo de Vida de escolares brasileiros. Nutr Clín y Diet Hosp. 2016;36(1):17-23. https://doi.org/10.12873/ 361 parente.

52. Brasil LMP, Fisberg M, Maranhão HS. Excesso de peso de escolares em região do Nordeste Brasileiro: contraste entre as redes de ensino pública e privada. Rev Bras Saude Matern Infant. 2007;7(4):405-12. https://doi.org/10. 1590/S1519-38292007000400008

53. Giugliano R, Carneiro EC. Fatores associados à obesidade em escolares. J Pediatr. 2004:80(1):17-22. https://doi.org/10.2223/1128.

54. Monego ET, Jardim PCBV. Determinantes de Risco Para doenças cardiovasculares em escolares. Arq Bras Cardiol. 2006;87(1):37-45. https:// doi.org/10.1590/S0066-782X2006001400006.

55. Baruki SBS, Rosado LEFPL, Rosado GP, et al. Associação entre estado nutricional e atividade física em escolares da Rede municipal de Ensino em Corumbá-MS. Rev Bras Med Esporte. 2006;12(2):90-4. https://doi.org/10. 1590/S1517-86922006000200007.

56. Pereira FN, Oliveira JR, Zollner CC, et al. Body weight perception and associated factors in students. J Human Growth Dev. 2013;23(2):170-6. https://doi.org/10.7322/jhgd.69504.

57. Correa TAF, Cogni R, Cintra RMGC. Estado nutricional e consumo alimentar de adolescentes de uma escola municipal de Botucatu, SP. Rev SimbioLogias. 2008;1(1):130-44. https://doi.org/10.32905/19833253.2008.01.01p130.

58. Ribeiro RQC, Lotufo PA, Lamounier JA, et al. Fatores adicionais de Risco cardiovascular associados ao excesso de peso em crianças e adolescentes: o estudo do coração de Belo Horizonte. Arq Bras Cardiol. 2006;86(6):408-18. https://doi.org/10.1590/S0066-782X2006000600002.

59. Leal VS, Lira PIC, Oliveira JS, et al. Excesso de peso em crianças e adolescentes no Estado de Pernambuco, Brasil: prevalência e determinantes. Cad Saúde Pública. 2012;28(6):1175-82. https://doi.org/10.1590/S0102$311 \times 2012000600016$

60. Campos LA, Leite AJM, Almeida PC. Prevalência de sobrepeso e obesidade em adolescentes escolares do município de Fortaleza, Brasil. Rev Bras Saude Matern Infant. 2007;7(2):183-90. https://doi.org/10.1590/S151938292007000200009

61. Pinto ICS, Arruda IKG, Diniz AS, et al. Prevalência de excesso de peso e obesidade abdominal, segundo parâmetros antropométricos, e associação com maturação sexual em adolescentes escolares. Cad Saúde Pública. 2010; 26(9):1727-37. https://doi.org/10.1590/S0102-311X2010000900006.

62. Pawellek I, Grote $\mathrm{V}$, Theurich $\mathrm{M}$, et al. Factors associated with sugar intake and sugar sources inEuropean children from 1 to 8 years of age. Eur J Clin Nutr. 2017:71:25-32. https://doi.org/10.1038/ejcn.2016.206.

63. Kelishadi R, Azizi-Soleiman F. Controlling childhood obesity: a systematic review on strategies and challenges. J Res Med Sci. 2014;19(10):993-1008 https://www.ncbi.nlm.nih.gov/pmc/articles/PMC4274579/ Acess 05 Sep 2019.

64. Lipsky LM, lannotti RJ. Associations of television viewing with eating behaviors in the 2009 health behaviour in school-aged children study. Arch Pediatr Adolesc Med. 2012;166(5):465-72. https://doi.org/10.1001/ archpediatrics.2011.1407.

65. Lee IM, Shiroma EJ, Lobelo F, et al. Effect of physical inactivity on major non-communicable diseases worldwide: an analysis of burden of disease and life expectancy. Lancet. 2012;380(9838):219-29. https://doi.org/10.1016/ S0140-6736(12)61031-9.

66. Lipsky LM, lannotti RJ. Associations of television viewing with eating behaviors in the2009 health behaviour in school-aged children study. Arch Pediatr Adolesc Med. 2012;166(5):465-72. https://doi.org/10.1001/ archpediatrics.2011.1407.

67. Falbe J, Rosner B, Willet WC, et al. Adiposity and different types of screen time. Pediatrics. 2013;132(6):e1497-505. https://doi.org/10.1542/peds.20130887. 
68. Dietz WH, Baur LA, Hall K, et al. Management of obesity: improvement of health-care training and systems for prevention and care. Lancet. 2015; 385(9986):2521-33. https://doi.org/10.1016/50140-6736(14)61748-7.

69. Plachta-Danielzik S, Landsberg B, Lange D, et al. Eight-year follow-up of school-based intervention on childhood overweight-the Kiel obesity prevention study. Obes Facts. 2011:4(1):35-43. https://doi.org/10.1159/ 000324552.

70. Fagen MC, Asada Y, Welch S, et al. Policy, systems, and environmentally oriented school-based obesity prevention: opportunities and challenges. J Prev Interv Community. 2014;42(2):95-111. https://doi.org/10.1080/ 10852352.2014.881175.

71. Sabin MA, Kiess W. Childhood obesity: current and novel approaches. Best Pract Res Clin Endocrinol Metab. 2015;29(3):327-38. https://doi.org/10.1016/j. beem.2015.04.003.

72. Zanirati VF, Lopes ACS, Santos LC. Contribuição do turno escolar estendido Para o perfil alimentar e de atividade física entre escolares. Rev Panam Salud Publica. 2014;35(1):38-45 http://iris.paho.org/xmlui/handle/123456789/ 8543 Acess 05 Sep 2019.

73. Assis MM, Leite MA, Côrtes AJ, et al. Excesso de peso, ambiente percebido e privação social: um estudo da percepção de pais ou responsáveis. Rev Paul Pediatr. 2018;36(4):466-73. https://doi.org/10.1590/1984-0462/;2018;36;4; 00011.

74. Assis MM, Leite MA, Carmo ASD, et al. Food environment, social deprivation and obesity among students from Brazilian public schools. Public Health Nutr. 2019;22(11):1920-7. https://doi.org/10.1017/S136898001800112X.

75. Castro A, Lopes RE. A escola de tempo integral: desafios e possibilidades. Ensaio: Aval Pol Públ Educ. 2011;19(71):259-82. https://doi.org/10.1590/ S0104-40362011000300003.

76. Lülfs-Baden F, Spiller A. Students' perceptions of school meals: a challenge for schools, school-meal providers, and policymakers. J Food. 2009;20(1):3146. https://doi.org/10.1111/j.1748-0159.2008.00121.x.

77. World Health Organization. Population-based prevention strategies for childhood obesity: report of a WHO forum and technical meeting, Geneva, 15-17 December 2009. Geneva: WHO; 2010. Available at https:// wwwwhoint/dietphysicalactivity/childhood/child-obesity-engpdf Acessed 30 May 2017.

78. World Health Organization. Population-based approaches to childhood obesity prevention. Geneva: WHO; 2012. Available at https:/apps.who.int/ iris/bitstream/handle/10665/80149/9789241504782_eng.pdf?sequence=1. Acessed 30 May 2017.

79. Brasil. Ministério da Saúde. Perspectivas e desafios no cuidado as pessoas com obesidade no SUS: resultados do Laboratório de Inovação no manejo da obesidade nas Redes de Atenção à Saúde. Brasília: Ministério da Saúde: 2014. Available at http://bvsms.saude.gov.br/bvs/publicacoes/perspectivas desafios_cuidado_pessoas_obesidade.pdf Acessed 17 Mar 2018.

80. Medeiros CCM, Cardoso MAA, Pereira RAR, et al. Estado nutricional e hábitos de Vida em escolares. Rev Bras Crescimento Desenvolv Hum. 2011;21(3): 789-97. https://doi.org/10.7322/jhgd.20031.

81. Carmo AS, Assis MM, Cunha CF, et al. The food environment of Brazilian public and private schools. Cad Saúde Pública. 2018;34(12):e00014918. https://doi.org/10.1590/0102-311×00014918.

82. Fundo Nacional de Desenvolvimento da Educação. Resolução n 26,17 de junho de 2013. Brasília: Diário Oficial da União; 2013. Available at https:// www.fndegovbr/indexphp/acesso-a-informacao/institucional/legislacao/ item/4620-resolu\%C3\%A7\%C3\%A3o-cd-fnde-n\%C2\%BA-26,-de-17-de-junhode-2013 Acessed 20 Aug 2019.

83. Cairns K. Connecting to food: cultivating children in the school garden. Children's Geographies. 2017;15(3):304-18. https://doi.org/10.1080/14733285. 2016.1221058

84. Beckman LL, Smith C. An evaluation of inner-city youth garden program participants' dietary behavior and garden and nutrition knowledge. J Agric Educ. 2008;49(4):1-24. https://doi.org/10.5032/jae.2008.04011.

85. Morgan PJ, Warren JM, Lubans DR, et al. The impact of nutrition education with and without a school garden on knowledge, vegetable intake and preferences and quality of school life among primary-school students. Public Health Nutr. 2010;13(11):1931-40. https://doi.org/10.1017/ S1368980010000959.

86. Cotugna N, Manning CK, DiDomenico J. Impact of the use of produce grown in an elementary school garden on consumption of vegetables at school lunch. J Hunger Environ Nutr. 2012;7(1):11-9. https://doi.org/10.1080/ 19320248.2012 .649668
87. Roche E, Kolodinsky JM, Johnson RK, et al. School gardens may combat childhood obesity. Choices. 2017;32(1):1-6. https://doi.org/10.22004/ag.econ 253379 .

88. Guililland JA, Rangel CY, Healy MA, et al. Linking childhood obesity to the built environment: a multi-level analysis of home and school neighbourhood factors associated with body mass index. Can J Public Health. 2012;103(9 Suppl 3):S15-21. https://doi.org/10.1007/BF03403830.

89. Wake M, Lycett K, Clifford SA, et al. Shared care obesity management in 310 year old children: 12 month outcomes of hop SCOTCH randomised trial. BMJ. 2013;346:f3092. https://doi.org/10.1136/bmj.f3092.

90. Muckelbauer R, Libuda L, Clausen $K$, et al. A simple dietary intervention in the school setting decreased incidence of overweight in children. Obes Facts. 2009;2(5):282-5. https://doi.org/10.1159/000229783.

91. Reihner T, Widhalm K, l'Allemand D, et al. Two-year follow-up in 21,784 overweight children and adolescents with lifestyle intervention. Obesity. 2009;17(6):1196-9. https://doi.org/10.1038/oby.2009.17.

92. Taveras EM, Marshall R, Kleinman KP, et al. Comparative effectiveness of childhood obesity interventions in pediatric primary care a clusterrandomized clinical trial. JAMA Pediatr. 2015;169(6):535-42. https://doi.org/ 10.1001/jamapediatrics.2015.0182.

93. Fox MK, Dodd AH, Wilson A, et al. Association between school food environment and practices and body mass index of US public school children. J Am Diet Assoc. 2009;109(2 Suppl):S108-17. https://doi.org/10. 1016/j.jada.2008.10.065.

94. Li J, Hooker NH. Childhood obesity and schools: evidence from the National Survey of Children's health. J Sch Health. 2010;80(2):96-103. https://doi.org/ 10.1111/j.1746-1561.2009.00471.x.

95. Heelan KA, Bartee RT, Nihiser A, et al. Healthier school environment leads to decreases in childhood obesity: the Kearney Nebraska Story. Child Obes. 2015;11(5):600-7. https://doi.org/10.1089/chi.2015.0005.

96. Welker E, Lott M, Story M. The school food environment and obesity prevention: progress over the last decade. Curr Obes Rep. 2016;5:145-55. https://doi.org/10.1007/s13679-016-0204-0.

97. Cacau LAP, Carvalho CVO, Pin AS, et al. Reference values for the 6-min walk distance in healthy children age 7 to 12 years in Brazil: Main results of the TC6minBrasil multi-center study. Respir Care. 2018;63(3):339-46. https://doi. org/10.4187/respcare.05686.

\section{Publisher's Note}

Springer Nature remains neutral with regard to jurisdictional claims in published maps and institutional affiliations.

Ready to submit your research? Choose BMC and benefit from:

- fast, convenient online submission

- thorough peer review by experienced researchers in your field

- rapid publication on acceptance

- support for research data, including large and complex data types

- gold Open Access which fosters wider collaboration and increased citations

- maximum visibility for your research: over $100 \mathrm{M}$ website views per year

At $\mathrm{BMC}$, research is always in progress.

Learn more biomedcentral.com/submissions 